\title{
Calculation of the Rate of Coagulation of Hydrophobic Colloids in the Non-Steady State
}

\author{
G. J. ROEBERSEN AND P. H. WIERSEMA \\ Van't Hoff Laboratory, University of Uirecht, Transitorium III, Padualaan 8, Utrecht, The Netherlands
}

Received November 9, 1973; accepted February 28, 1974

\begin{abstract}
In accurate coagulation measurements, the observed coagulation rate should be extrapolated to time zero to find the rate of formation of doublets from singlet particles. In the theoretical calculation of coagulation rates, generally a steady state is assumed. At the onset of coagulation, however, a transient state is present. The results are given of numerical computations of the transient-state coagulation rate for the cases of Van der Waals attraction only and of Van der Waals attraction combined with double layer repulsion. The duration of the non-steady state is about the same as the one calculated by Von Smoluchowski for the case of no long-range interaction. It is expected that no transient effects will be found in classical coagulation experiments, but they may interfere with rapid (stopped-flow) measurements of the rate of rapid coagulation.
\end{abstract}

\section{INTRODUCTION}

During the coagulation of a monodispersed hydrophobic colloid, doublet particles are formed and these react further to form triplets, quadruplets, etc. The presence of multiplet particles markedly complicates the picture of the reaction kinetics. Although Von Smoluchowski (1) has worked out an approximate theory for the rate of coagulation in a system of singlet and multiplet particles, for a quantitative test of coagulation theory one should preferably use experimental information concerning the formation of doublets from singlets. Therefore the rate of coagulation, determined in an originally monodisperse sol with spherical particles, should be extrapolated to time zero to find the true bimolecular rate constant. The extrapolation can be improved by rapidly mixing the sol and the coagulating agent and measuring immediately afterwards, and by working with small sol concentrations.

Then, however, a new difficulty arises. Current theories on the rate of coagulation are based on the presence of a steady state, but immediately after the beginning of the coagulation process a transient state occurs. An analytical calculation of the rate of coagulation during this initial state has already been performed by Von Smoluchowski for the case of no long-range interaction between the particles (1). The conclusion is that a marked deviation of the steady-state rate exists only during a small fraction of the half-time of coagulation. It is, however, not certain a priori that this conclusion is valid when there are interaction forces between the particles (e.g., Van der Waals attraction and double layer repulsion). In the latter case an analytical solution is not known. In this paper, we present the results of a numerical computation for the non-steady-state coagulation for a number of interaction types. We also take into account the effect of hydrodynamic interaction between approaching particles $(2,3)$.

\section{STATEMENT OF THE PROBLEM}

We used a model for the coagulation process that is the same as the one devised by Von 
Smoluchowski (1) and extended by Fuchs (5). All particles are spheres with radius $a$. One particle is considered to be the "central" one and the origin of the system of coordinates is placed at its center. Any other particle, coming in contact with this one, will permanently stick to it. So no (centers of) particles can be present within a sphere of radius $2 a$ around the central particle. The force, acting upon a particle at distance $r$ from the central one, is denoted by $F(r)$; its sign is positive at repulsion. The process of coagulation is now treated in the model as a diffusion process in a field of force, governed by the equation:

$$
\frac{\partial \nu}{\partial t}=\frac{1}{r^{2}} \frac{\partial}{\partial r}\left\{r^{2} D \frac{\partial \nu}{\partial r}-r^{2} D \frac{F(r)}{k T} \nu\right\} .
$$

Here $\nu$ denotes the number of particles per unit volume; $t$ is time; $r$ is the distance from the center of a particle to the central particle's center; $D$ is the diffusion coefficient of a particle with respect to another (viz., the central one), i.e., twice the diffusion coefficient of a single particle; $k$ is Boltzmann's constant; and $T$ is the absolute temperature. In stating Eq. [1] it is assumed that Einstein's relation (mobility of the particle $=D / k T$ ) is valid. We use the boundary conditions applied by Von Smoluchowski and Fuchs:

$$
\begin{gathered}
\text { At } t=0, \nu=\nu_{0} \text { for } 2 a<r<\infty ; \\
\text { at } t>0, \nu=0 \text { for } \quad r=2 a ; \\
\text { at } t \geqslant 0, \nu=\nu_{0} \text { for } \quad r \rightarrow \infty .
\end{gathered}
$$

Here $p_{0}$ denotes the initial value of the particle concentration. Solution of Eqs. [1]-[4] gives the particle concentration as a function of distance to the central particle and time. From this concentration, the rate of coagulation is calculated by considering the number of particles crossing a sphere, concentric with the central particle, in unit time, called the "flux" $J$; in accordance with Eq. [1], $J$ equals

$$
J(r)=4 \pi\left\{r^{2} D \frac{\partial \nu}{\partial r}-r^{2} D \frac{F(r)}{k T} \nu\right\} .
$$

The value of the flux for $r \rightarrow 2 a$ gives the number of particles that reach the central particle in unit time. The rate of coagulation, i.e., the number of primary particles disappearing from unit volume in unit time, is now found by multiplying this value of the flux by the particle concentration (4).

For special cases, an analytical solution of Eqs. [1]-[4] is known. Von Smoluchowski has already found the solution for the case of no force of interaction between the particles, i.e., $F(r) \equiv 0$ :

where

$$
\nu(r, t)=\nu_{0}\left[1-\frac{2 a}{r} \operatorname{erfc}\left\{\frac{r-2 a}{2 \sqrt{(D t)}}\right\}\right],
$$

$$
\operatorname{erfc} x=\frac{2}{\sqrt{\pi}} \int_{x}^{\infty} \exp \left(-z^{2}\right) d z \text {. }
$$

For large times the argument of the complementary error function, erfc, becomes very small, so that the value of this function approaches 1:

$$
\nu(r, t \rightarrow \infty)=\nu_{0}\left(1-\frac{2 a}{r}\right) .
$$

This is the so-called steady-state solution, in which the particle concentration in any volume element remains constant. This result can directly be found by setting $\partial \nu / \partial t=0$ in Eq. [1]. Differentiation of Eq. [6] with respect to distance, and substitution of the result into Eq. [5] (with $F(r) \equiv 0$ ) gives

$$
J(r \rightarrow 2 a)=4 \pi \cdot 2 a D \nu_{0}\left\{1+\frac{2 a}{\sqrt{(\pi D t)}}\right\}
$$

which in the steady state $\left(l \gg a^{2} / D\right)$ becomes

$$
J=8 \pi a D \nu_{0}
$$

In the latter case, the flux is independent of position, as the particle concentration remains constant everywhere (cf., Ref. 4).

Assuming the presence of a steady state $(\partial \nu / \partial t \equiv 0)$ Fuchs has solved Eqs. [1]-[4] for the case of interaction forces between particles (5). 
The purpose of the present work is to find solutions of the complete set of Eqs. [1]-[4], to see whether a steady state is reached and if so, after how much time. Two kinds of forces between the particles are considered: Van der Waals forces and electrostatic repulsion forces. For the Van der Waals attraction, we used the formula given by Hamaker (6) for two equal spheres, from which the formula for the force is found by differentiation

$$
F_{A}(s)=\frac{-32 A}{3 a s^{3}\left(s^{2}-4\right)^{2}} .
$$

Here $A$ denotes the Hamaker constant, and $s=r / a$. This equation does not take into account the retardation effect; this is justified in our case because this effect is important only at large distances, with the result that its contribution to the rate of coagulation is negligible. Apart from the retardation effect, the validity of this and related formulas has been the subject of much recent discussion (7); but, up to the present, no alternative equation for two spheres has been proposed. ${ }^{1}$

A number of approximate equations for the double layer repulsion force (8-11) have been compared with numerically calculated results $(10-13) .{ }^{2}$ Since both the force and its derivative are used in our computations, an approximate equation should represent the exact values as accurately as possible. For the case of constant potential of the particles, one of the best approximations for all interparticle distances and a large range of potentials and electrolyte concentrations appeared to be given by Derjaguin's equation (9):

$$
F_{R}(s)=\frac{\epsilon \psi_{\delta}^{2} \tau}{2} \cdot \frac{\exp \{-\tau(s-2)\}}{1+\exp \{-\tau(s-2)\}},
$$

where $\epsilon$ denotes the dielectric constant of the

1 Note added in proof. Very recently, formulas for the van der Waals interaction between two spheres were given by SMith, E. R., Mitchell, D. J., AND NinhaM, B. W., J. Colloid Interface Sci. 45, 55 (1973).

2 This work was performed by $\mathrm{J}$. J. Vossen in our laboratory. liquid, $\psi_{\delta}$ is the electrostatic potential in the Outer Helmholtz Plane, and $\tau=\kappa a$, where $\kappa$ is the Debye-Hückel reciprocal length. This equation, however, is not valid for very large potentials. We did not perform calculations for constant charge, as no suitable analytical approximation for this case is available, and as we did not expect the results to differ much from those for constant potential.

As to the diffusion coefficient, we not only worked out the "classical" case, in which it is considered to be constant, but also the more realistic case, in which it varies with distance between the particles as a result of the hydrodynamic interaction $(2,3)$. In the latter case we used the approximate equation (3):

$$
D(s)=D_{0} \cdot \frac{6 s^{2}-20 s+16}{6 s^{2}-11 s}
$$

where $D_{0}$ denotes the diffusion coefficient of a particle in an infinitely dilute solution.

\section{METHOD OF COMPUTATION}

Equation [1] and the boundary conditions [2]-[4] were transformed into a dimensionless form by the substitutions:

$$
\begin{aligned}
c & \equiv \nu / \nu_{0}, \\
y & \equiv a / r \equiv 1 / s, \\
\theta & \equiv D_{0} t / a^{2}, \\
\beta(y) & \equiv D_{0} / D(y), \\
\varphi(y) & \equiv a F(r) / k T .
\end{aligned}
$$

Equation [1] becomes

$$
\frac{\partial c}{\partial \theta}=y^{4} \times \frac{\partial}{\partial y}\left[\frac{1}{\beta(y)}\left\{\frac{\partial c}{\partial y}+\frac{\varphi(y) c}{y^{2}}\right\}\right],
$$

with the boundary conditions:

$$
\begin{aligned}
& c(y, \theta=0)=1 \text { for } 0<y<\frac{1}{2}, \\
& c\left(y=\frac{1}{2}, \theta\right)=0 \text { for } \theta>0, \\
& c(y=0, \theta)=1 \text { for } \theta \geqslant 0 .
\end{aligned}
$$

An advantage of the use of $y$ as an independent variable is that it varies over a finite interval, 
which greatly facilitates the numerical computations. Equation [14] is a linear partial differential equation of parabolic type. The boundary value problem, constituted by this equation and conditions [15]-[17], was solved by use of the method of Crank and Nicolson (14).

An equation for the dimensionless flux is obtained by substituting Eq. [13] into Eq. [5]:

$$
\frac{J}{4 \pi a D_{0} \nu_{0}} \equiv N=\frac{-1}{\beta(y)}\left\{\frac{\partial c}{\partial y}+\frac{\varphi(y) c}{y^{2}}\right\} .
$$

Taking into account that in this case $D_{0}$ is a mutual diffusion coefficient, the value of the dimensionless flux $N$ for $r \rightarrow 2 a$ (i.e., $y \rightarrow 0.5$ ), appears to be equal to the dimensionless rate constant defined in Ref. 3. In obtaining this limiting value of $N$ from the r.h.s. of Eq. [18], we had to deal with the problem that $\varphi(y)$ approaches minus infinity, while $c$ approaches zero in this limit (although the value of the complete r.h.s. expression remains finite). Therefore $N$ was calculated from the values of the separate terms for $y_{1} \equiv 0.5-\Delta y$, where $\Delta y$ is the interval used in the numerical computation. Since the approximation $(\partial c / \partial y)_{y=y_{1}}$ $=\Delta c / \Delta y$ proved to be inaccurate, we computed $(\partial c / \partial y)_{y=y_{1}}$ from the series

$$
c(y)=\sum_{j=0}^{n} b_{j} y^{j} \quad(n \leqslant 5) .
$$

The coefficients, $b_{j}$, of this series were obtained by applying a least-squares method to the computed values of $c(y)$. This method was checked by varying the number, $n$, of terms in the series, and the number of points $(c, y)$ used in the computation. Usually, the relative variation in the dimensionless rate constant, produced this way, did not exceed $2 \%$.

Any numerical method introduces a discretization error into the results of the computation. By refining the grid (dimensionless distance and/or dimensionless time) the accuracy of the results was checked. In the cases of no interaction (Von Smoluchowski) and of Van der Waals interaction only (where the force varies monotonously with distance), there appeared to be little influence of mesh size. In the case of double layer repulsion together with Van. der Waals attraction, where the force has a maximum and a minimum, we found a stronger influence of mesh size on the obtained values of the concentration and the coagulation rate. Generally, halving the mesh size (for the dimensionless distance) improved the results sharply, but for a number of cases the limitation of the computer memory prevented the production of accurate results by this method.

Our computer program was written in ALGOL 60. We performed most of the computations on the Electrologica X8-computer of the Academic Computer Centre of Utrecht; later some computations were done on its new CYBER 73-26. The text of our computer program, together with an explanation of its use, can be obtained from the authors on request. The values of the interaction force and its derivative are generated by subroutines in the program; an evaluation for other types of interaction is possible by either changing the subroutines or giving numerical values for each grid point.

\section{RESULTS}

The "relaxation time," $t_{r}$, is defined here as the time, corresponding to $\theta=1$, i.e.,

$$
t_{\mathrm{r}}=a^{2} / D_{0}
$$

First, the Von Smoluchowski case (no interaction between the particles) was examined as a "test case" for the numerical method. Complete agreement between the numerical and the analytical results (Eqs. [6] and [8]) was obtained.

\section{(a) Rapid Coagulation}

When only Van der Waals attraction acts between the particles, "rapid coagulation" occurs. Relevant values of the Hamaker constant are between about $10^{-19}$ and a few times $10^{-22} \mathrm{~J}(7)$; we performed calculations for $A=10^{-19}, 10^{-20}$, and $10^{-21} \mathrm{~J}$ and a temperature 
of about $300 \mathrm{~K}$. The results are shown in Figs. 1 and 2. The constant $B \equiv A / 12 k T$; at $300 \mathrm{~K}, A$ is about $5 B \times 10^{-20} \mathrm{~J}$.

\section{(b) Slow Coagulation}

For these computations we chose a few cases, where the coagulation rate is a considerable fraction of the rate of rapid coagulation, i.e., the delay factor $W$ is smaller than 100 . The results are shown in Figs. 3, 4, and 5. The constant $C=\epsilon a \psi_{\delta}^{2} / 2 k T$ (cf. Eq. [11]). As an example of actual systems, corresponding to the given parameters we note that the case $B=0.2, C=30$ corresponds to particles with Hamaker constant $10^{-20} \mathrm{~J}$ (e.g., silica particles) of radius $50 \mathrm{~nm}$ and potential $\psi_{\delta}=24 \mathrm{mV}$. The $\tau=35$ case corresponds to a concentration of $45.7 \mathrm{mM}, \tau=25$ to a concentration of $23.3 \mathrm{mM}$ uni-univalent electrolyte. The case $B=2, C=90, \tau=20$ corresponds to particles with Hamaker constant $10^{-19} \mathrm{~J}$ (e.g., metal particles) of radius $150 \mathrm{~nm}$ and potential $\psi_{\delta}=24 \mathrm{mV}$ in a 1.66 $\mathrm{m} M$ solution of uni-univalent electrolyte. The $\tau=16$ case corresponds to an electrolyte concentration of $1.06 \mathrm{mM}$.
The values of the dimensionless rate constant $N$ at 100,000 times the relaxation time are not equal to the steady-state values, calculated numerically according to Fuchs; this is due to discretization errors from the numerical computation for the transient state. The difference between $N$ at $\theta=100,000$ and Fuchs' steady-state value decreases strongly when the grid is refined. The values, shown in the figures are produced with the finest grid allowed by the computer memory ( 1280 points). An interesting feature of four of the given cases is that the rate constant as a function of time shows a relative minimum and a relative maximum. This can be explained as follows. The steady decline of the rate constant in the beginning of the coagulation process is counteracted by the building up of a concentration peak at the place of the secondary minimum in the interaction energy (cf., Fig. 6). This concentration peak serves as a secondary source of particles that will coagulate in the primary minimum, and hence the coagulation rate increases again. After some time, the concentration peak has reached its maximum value; afterwards, it decreases slowly to its

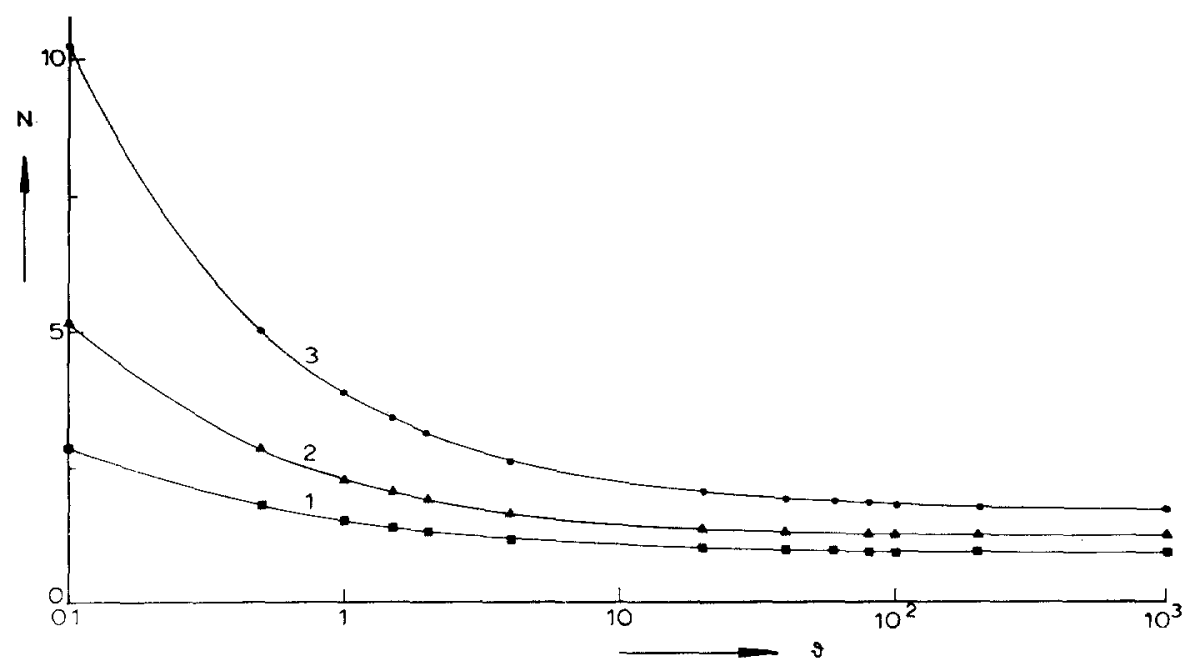

FIG. 1. Dimensionless rate constant for rapid coagulation as a function of dimensionless time. $N=J /$ $4 \pi a D_{0} \nu_{0} ; \theta=t D_{0} / a^{2}$. Hydrodynamic correction is included.
(1) $B=0.02 \quad\left(A=10^{-21} \mathrm{~J}\right.$ at $\left.25^{\circ} \mathrm{C}\right)$;
(2) $B=0.2 \quad\left(A=10^{-20} \mathrm{~J}\right.$ at $\left.25^{\circ} \mathrm{C}\right)$;
(3) $B=2 \quad\left(A=10^{-19} \mathrm{~J}\right.$ at $\left.25^{\circ} \mathrm{C}\right)$.

Journal of Colloid and Inierface Science, Vol. 49, No. 1, October 1974 


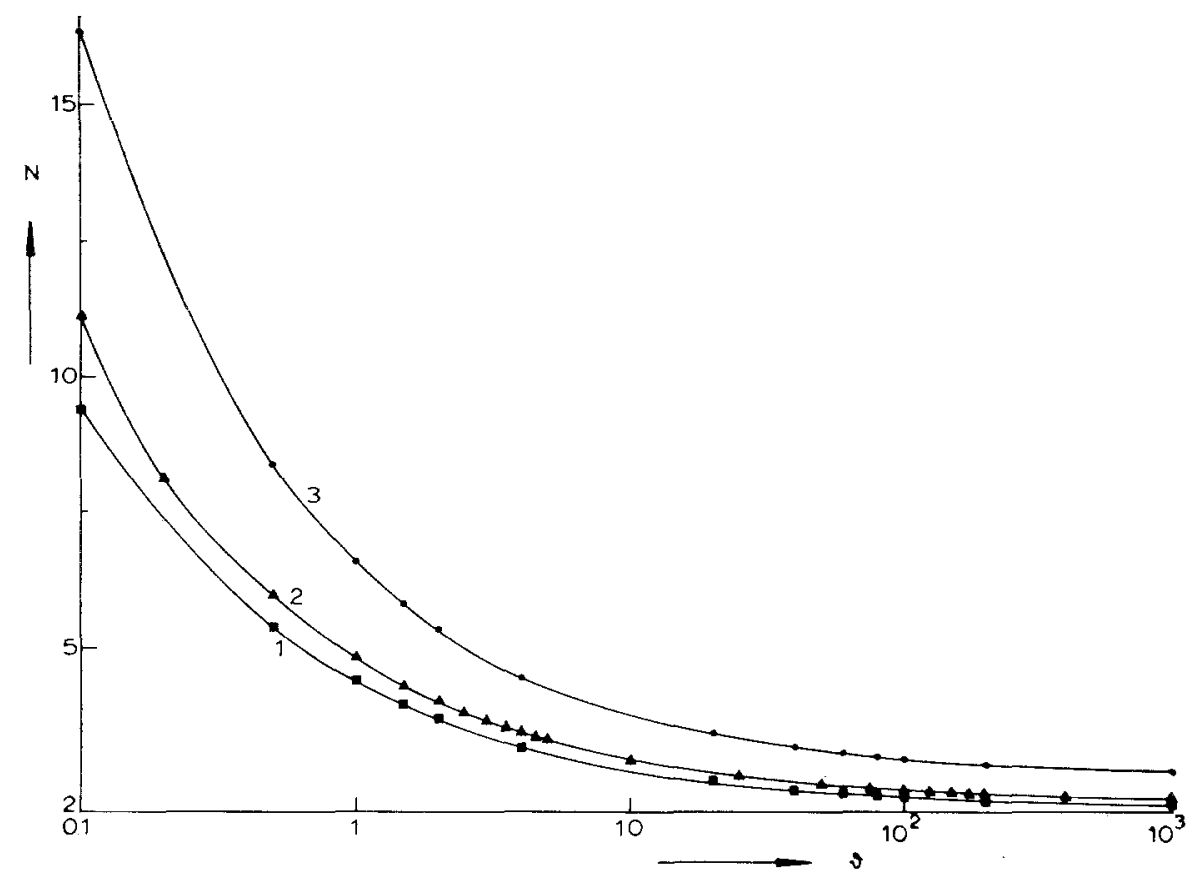

FIG. 2. Dimensionless rate constant for rapid coagulation as a function of dimensionless time. No hydrodynamic correction included. Numbers correspond to the same values of $B$ as in Fig. 1 .

steady-state value, as does the rate of As stated in Section III, it appeared to be coagulation. impossible to produce meaningful results when

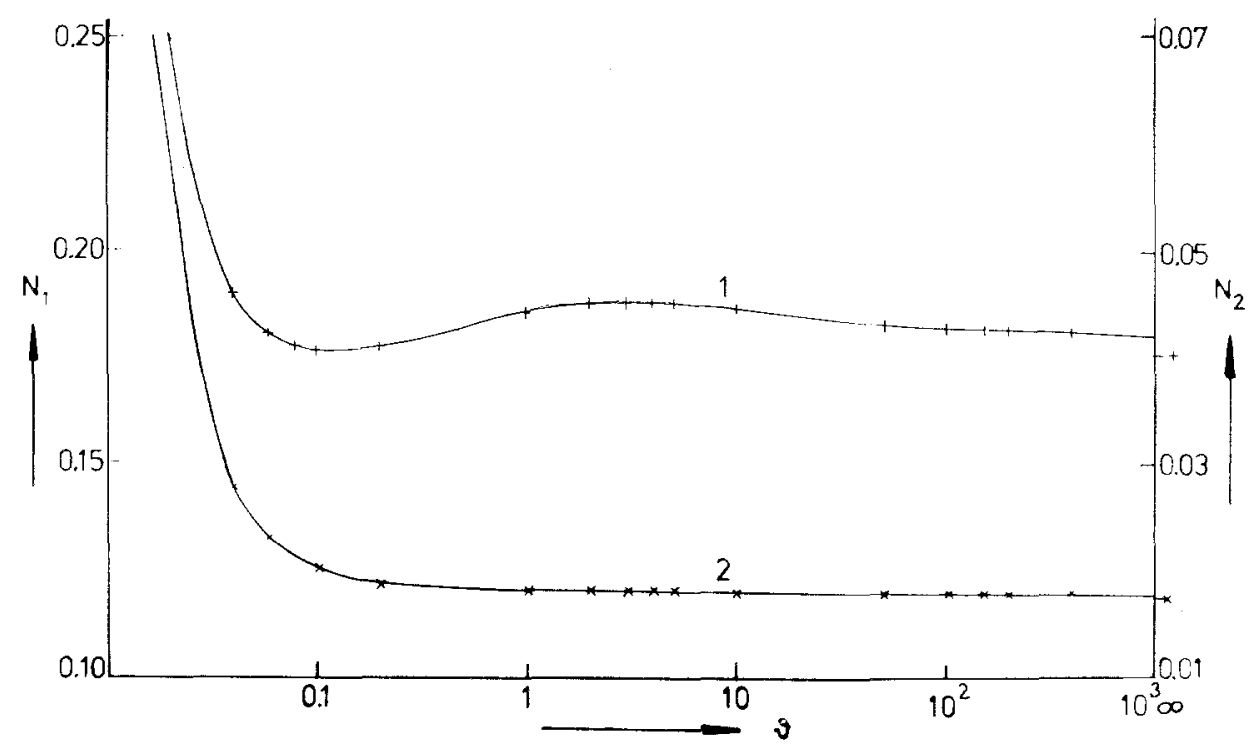

FIG. 3. Dimensionless rate constant for slow coagulation as a function of dimensionless time. Hydrodynamic correction is included. (1) $B=0.2, C=30, r=35 . B=A / 12 k T$; cf. Eq. [10]; $C=\epsilon a \psi_{\delta}^{2} /$ $2 k T$, and $\tau=\kappa a$; cf. Eq. [11]. The delay factor $W$ in the steady state $=6.60$. Read $N$ on left scale; (2) $B=0.2, C=30, \tau=25 . W$ (steady state) $=65.48$. Read $N$ on right scale. 


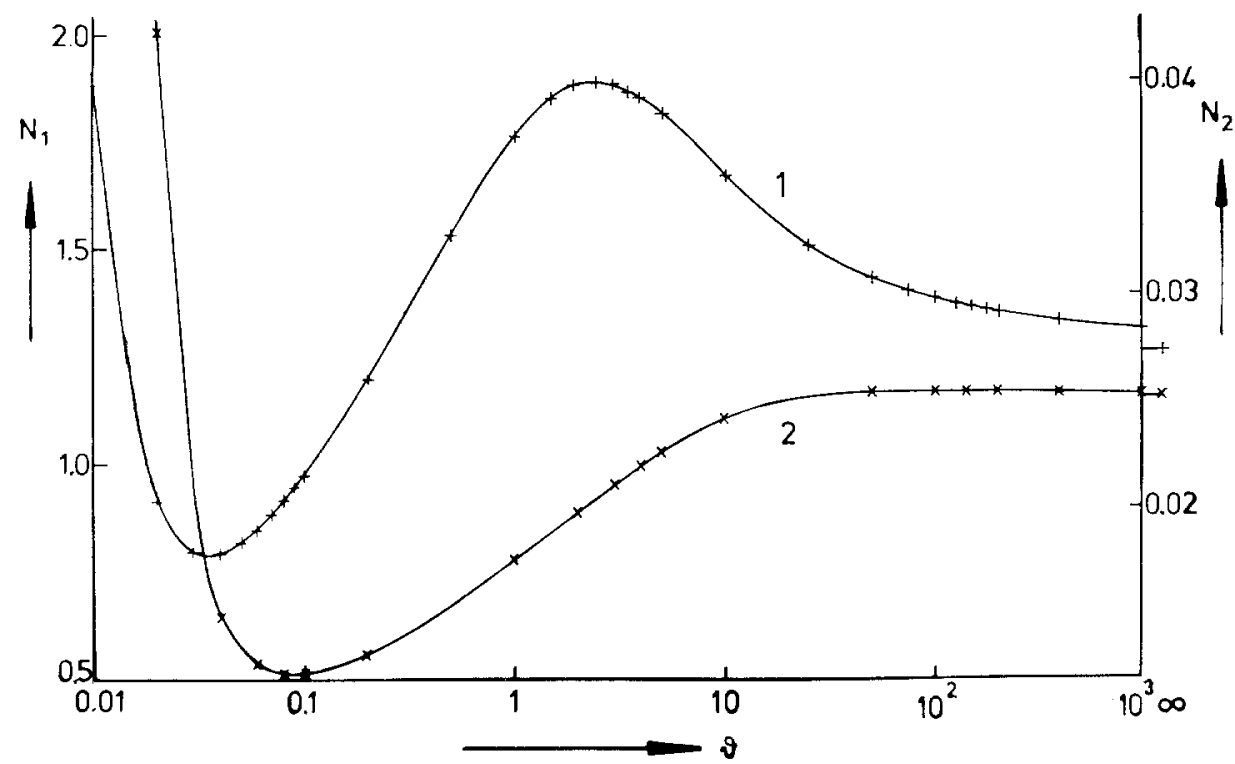

FIG. 4. Dimensionless rate constant for slow coagulation as a function of dimensionless time. Hydrodynamic correction is included. (1) $B=2, C=90, \tau=20 . W$ (steady state) $=1.30$. Read $N$ on left scale; (2) $B=2, C=90, \tau=16 . W$ (steady state) $=65.34$. Read $N$ on right scale.

a large maximum in the interaction force at a for $\theta>1$, the deviation decreases with inshort distance was present. But inspection of creasing repulsion force (cf. the case $B=0.2$, the figures shows definite trends in the size $C=30, \tau=25$, hydrodynamic correction with of the transient effect: The relative deviation the case $\tau=35$, other parameters unaltered:) from the steady-state rate, at a fixed dimen- Furthermore, the relative deviation from the sionless time, is generally less when electro- steady-state rate generally increases with static repulsion is present than when it is not; increasing Hamaker constant. This holds for

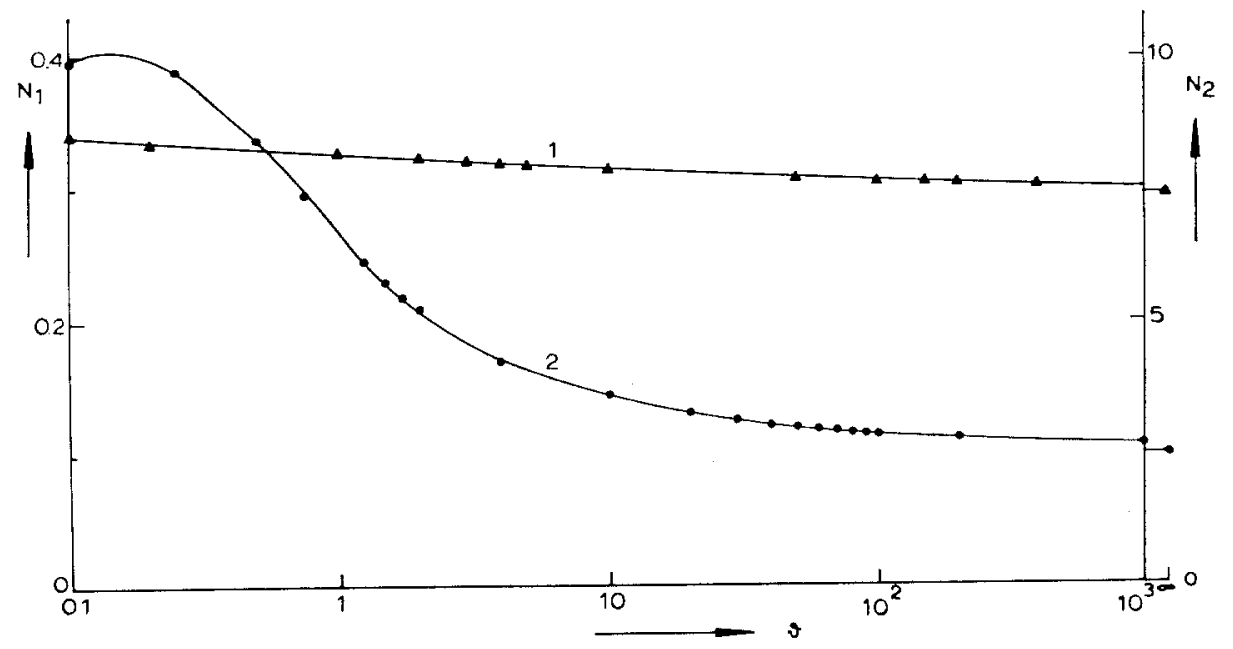

FIG. 5. Dimensionless rate constant for slow coagulation as a function of dimensionless time. No hydrodynamic correction included. (1) $B=0.2, C=30, \tau=25 . W$ (steady state) $=7.45$. Read $N$ on left scale; (2) $B=2, C=90, \tau=20 . W$ (steady state) $=1.06$. Read $N$ on right scale. 


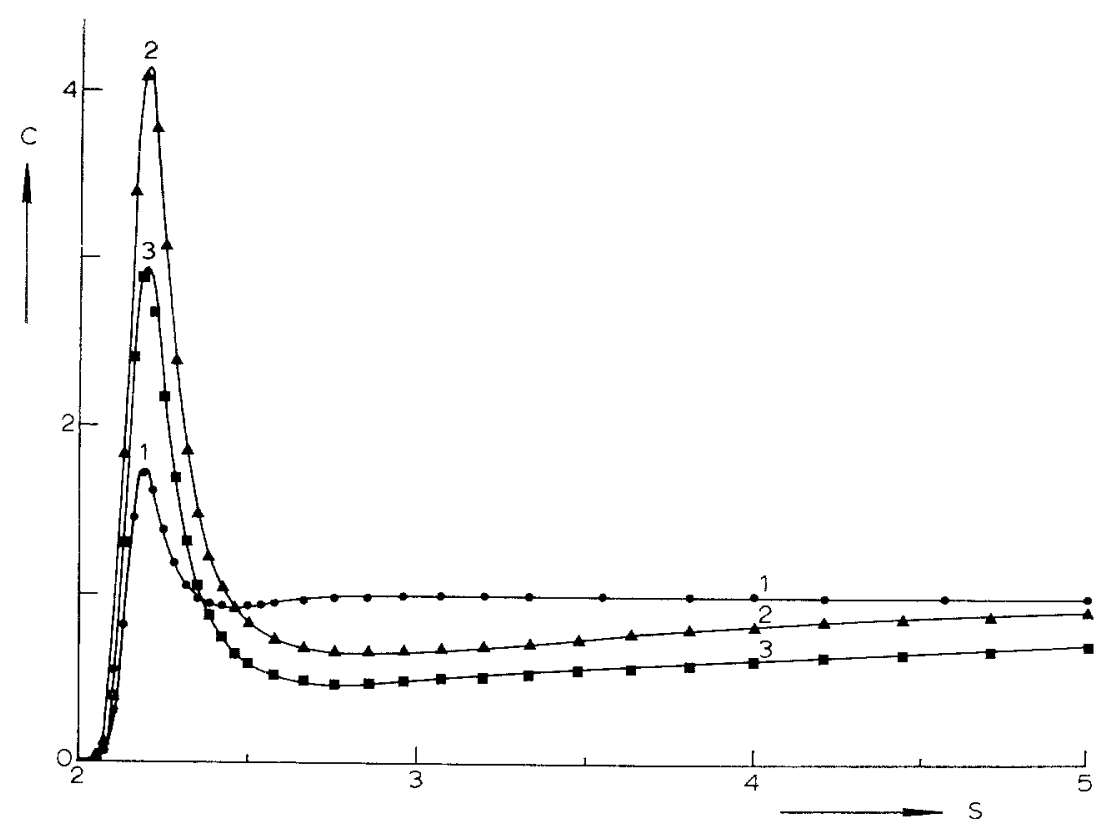

FIG. 6. Dimensionless particle concentration around a "central particle" as a function of dimensionless distance. $c=\nu / \nu_{0} ; s=r / a$. Hydrodynamic correction is included; $B=2, C=90, \tau=20$ [cf. Fig. 4, line (1)]: (1) $\theta=0.02$; (2) $\theta=5$; (3) $\theta=100000$.

both rapid and slow coagulation. This is a strong indication that in the cases of very stable sols (large $W$ ) and of slowly coagulating sols with a low Hamaker constant the transient effect is relatively even less important than in the reported cases.

\section{DISCUSSION}

From the results we concluded that generally-when Van der Waals attraction is present alone, or combined with double layer repulsion-the non-steady state occurs during a short time only, just as in the "pure Von Smoluchowski" case. The question of whether the non-steady state can be observed will be discussed.

In coagulation measurements there is a minimum time interval, say $\Delta t$, that is required to mix the sol and the coagulating agent and to obtain sufficient data afterwards; the value of $\Delta t$ depends on the experimental method. The non-steady state can be detected only if, at $t=\Delta t$, the rate of coagulation still differs appreciably from the steady-state value. From inspection of the figures, we see that this condition implies that $\theta<p$, where the value of $p$ is determined by the accuracy of the measurement and by the Van der Waals and double layer forces. For instance, when the coagulation rate must exceed the steady-state value by at least $10 \%$, the value of $p$ for a rapidly coagulating polystyrene latex $(B \approx 0.02)$ is about 20 (Fig. 1 ). From the definition of $\theta(\mathrm{Eq} .[13])$ we see that $\theta<p$ means $\left(t D_{0} / a^{2}\right)<p$. Since this condition must apply within $\Delta t$, we have

$$
a^{2} p / D_{0}>\Delta t
$$

Expressing the mutual diffusion coefficient $D_{0}$ by means of Stokes' law, we obtain

$$
a^{3}>\frac{k T}{3 \pi \eta} \cdot \frac{\Delta t}{p},
$$

which sets a lower limit to the particle radius at given experimental conditions; $\eta$ denotes the viscosity of the solvent. For water at $25^{\circ} \mathrm{C}$ and with $p=20,[22]$ reduces to 
$a>300(\Delta t)^{\frac{1}{3}}$, where $a$ is expressed in nanometers and $\Delta t$ in seconds.

There is, however, a second condition for detection of the transient state. The coagulation rate is measured by observing the rate of change of a certain quantity, such as number of particles or turbidity. To measure the coagulation rate, and a change of this rate with time, it is required that the relative change of the observed quantity be sufficient during the interval $\Delta t$. Assuming that the observed quantity is proportional to the particle concentration, $\nu$, we can express this condition as

$$
\frac{1}{\nu} \cdot \frac{d \nu}{d t} \cdot \Delta t>q
$$

where $q$ is again a number determined by the accuracy of the measurement; the value of $q$ is at least 0.05 . Since $(1 / \nu) \cdot(d \nu / d t)$ equals the "flux" $J$ (i.e., the frequency of collisions against one central particle) we obtain, using Eq. [9] as an approximation,

$$
8 \pi a D_{0} \nu_{0} \cdot \Delta t>q \text {. }
$$

The half-time of coagulation, according to Von Smoluchowski (1), is equal to $\Theta=\frac{1}{4} \pi a D_{0} \nu_{0}$, so this condition implies $\Delta t>\frac{1}{2} q \theta$.

The conditions [21] and [24] can be combined to give

$$
\varphi>q / 6 p
$$

where $\varphi=(4 / 3) \pi a^{3} \nu_{0}$ is the volume fraction of the colloid. Since we used Eq. [9], the condition [25] applies to rapid coagulation; for slow coagulation the right-hand side should be multiplied by the delay factor $W$. With $p=20$ and $q=0.05$, [25] sets a lower limit of $4.2 \times 10^{-4}$ to $\varphi$.

Returning to [22] we observe that the time interval, $\Delta t$, is at least $10 \mathrm{sec}$ for particle counting methods [e.g., flow ultramicroscope (15), Coulter counter (16); assuming that the coagulation is stopped artificially after $\Delta t]$ and also for classical turbidimetric methods. With $p=20$ and $\Delta t=10 \mathrm{sec},[22]$ implies $a>630 \mathrm{~nm}$, i.e., a particle diameter over
$1 \mu \mathrm{m}$. For particles of this size range, and $\varphi>4.2 \times 10^{-4}$ (condition [25]), the optical density of the sol is too high for accurate turbidimetric measurements. The Coulter counter is suitable for particles of this size. However, in any experimental method, orthokinetic coagulation occurring during the mixing of sol and electrolyte could very well cause additional complications for particles having a diameter over $1 \mu \mathrm{m}$ (17).

The turbidimetric stopped-flow method (18) can be used to measure coagulation rates. This reduces $\Delta t$ to about $0.1 \mathrm{sec}$. For this value of $\Delta t$, condition [21] implies $a>140 \mathrm{~nm}$. In this case, combination with the condition $\varphi>4 \times 10^{-4}$ will allow workable optical densities, but only for large wavelengths (near $800 \mathrm{~nm}$ ) and for a rather limited range of particle sizes (radius about $250 \mathrm{~nm}$ ) (19). ${ }^{3}$

We conclude this discussion with a few more general remarks on the diffusion model used in the computations, considering especially the question to which extent the application of the computed results is restricted by the use of the boundary conditions [2]-[4]. Strictly speaking, the assumption of a completely random initial particle distribution (Eq. [2]) is not correct for a sol with interacting particles, but for dilute sols this is not a serious problem. Moreover, convection during the mixing of sol and coagulating agent should provide a random distribution.

Equation [4], which assumes a constant concentration in the region far away from the central particle, looks unrealistic because, in a coagulating sol, the particle concentration decreases everywhere. The coagulation rate constant $k_{c}$ of a real sol, however, is calculated from the "bimolecular" coagulation rate $k_{c} \nu^{2}(t)$, where $\nu(t)$ is the time-dependent average particle concentration in the sol. In this

3 Experiments with the stopped-flow method in our laboratory (rapid coagulation of polystyrene latex) did not, up to the present, show transient effects. It is rather difficult to obtain the conditions, for which the result should be observable, and the accuracy of the apparatus is rather poor under these conditions. 
way the rate constant itself is calculated to a good approximation, even for advanced stages of the coagulation.

In our opinion, Eq. [3] is a more restricting boundary condition. The use of this "sink condition" implies that only particles that stick to each other are considered as coagulated, while an optical detection method may "see" a doublet when two particles are near to each other, but do not yet touch. Hence, the model cannot be applied to coagulation (or flocculation with polymers) into a secondary minimum. It would be interesting to replace Eq. [3] by

$$
J=0 \quad \text { at } \quad r=2 a \text { and } t \geqslant 0,
$$

which only implies that the particles cannot penetrate into each other. [This condition has already been mentioned by Von Smoluchowski (20) in a somewhat different context.] With Eq. [26] instead of [3], the complete partial differential equation [1] must be solved to obtain the coagulation rate at all. The simplification $\partial \nu / \partial t=0$ would in this case give only the final equilibrium state, which is a Boltzmann distribution of particles in the interaction field. When this field has a deep primary minimum, the final state corresponds to a completely coagulated sol.

\section{CONCLUSIONS}

When Van der Waals attraction and/or double layer repulsion are taken into account in the computations, the transient state occurs during about the same time as in the case of no long-range interaction, for which an analytical solution was known. Hydrodynamic interaction tends to diminish the duration of the transient state. The relative deviation from the steadystate rate during the transient stage is generally less, when electrostatic repulsion is present.

In classical coagulation rate measurements, no non-steady-state effects can be observed. Under certain circumstances, the transient state may just be observable in stopped-flow experiments on rapid coagulation.

\section{ACKNOWLEDGMENTS}

The authors wish to thank W. A. K. Maas and F. J. M. von der Linden (Academic Computer Centre Utrecht) for help and advice with the numerical computations. We thank Professor J. Th. G. Overbeek and J. W. Th. Lichtenbelt for useful discussions, and J. J. Vossen for his work on double layer repulsion formulas and a program for graphical representation of the results.

\section{REFERENCES}

1. Von Smoluchowski, M., Phys. Z. 17, 593 (1916).

2. Spielman, L. A., J. Colloid Interface Sci. 33, 562 (1970).

3. Honig, E. P., Roebersen, G. J., AND Wiersema, P. H., J. Colloid Interface Sci. 36, 97 (1971).

4. Overbeek, J. Th. G., in "Colloid Science," (H. R. Kruyt, Ed.), Vol. I, pp. 278-286. Elsevier, Amsterdam, 1952.

5. Fuchs, N. A., Z. Pllys. 89, 736 (1934).

6. Hanaker, H. C., Physica (Utrecht) 4, 1058 (1937).

7. VISSER, J., Advan. Colloid Interface Sci. 3, 331 (1972).

8. Verwey, E. J. W., and Overbeek, J. Th. G., "Theory of the Stability of Lyophobic Colloids." Elsevier, Amsterdam, 1948.

9. Derjaguin, B. V., Trans. Faraday Soc. 36, 203 (1940).

10. McCartney, L. N., and Levine, S., J. Colloid Interface Sci. 30, 345 (1969).

11. Honig, E. P., and Mur, P. M., J. Colloid Interface Sci. 36, 258 (1971).

12. Hoskin, N. E., and Levine, S., Philos. Trans. Roy. Soc. London, A248, 433 (1956).

13. Beld, G. M., Levine, S, and McCartney, L. N., J. Colloid Interface Sci. 33, 335 (1970).

14. See, e.g., Anrs, W. F., "Numerical Methods for Partial Differential Equations," pp. $50 \mathrm{ff}$. Nelson, London, 1969.

15. Derjagutn, B. V., and Koudryavtseva, N. M., in "The Law of Mass Action. A Centenary Volume" (O. Bastiansen, Ed.), p. 79. Universitetsforlaget, Oslo, 1964; also Sмith, A. L., Special Disc. Faraday Soc. 1, 203 (1970) (discussion remark).

16. Matthews, B. A., ANd Rhodes, C. T., J. Colloid Interface Sci. 28, 71 (1968); 32, 332, 339 (1970).

17. Overbeek, J. Tr. G., Ref. 4, pp. 289-292.

18. ReICH, R. M., Anal. Chem. 43, 12, 85A (1971).

19. Pangonis, W. J., Heller, W., and Jacobson, A., "Tables of Light Scattering Functions for Spherical Particles," p. 115. Wayne State University Press, Detroit, 1957.

20. Von Smoluchowski, M., Phys. Z. 17, 586 (1916). 